\title{
Nachdruck:
}

\section{Von der Universalität der Wissenschaftsgeschichte}

Reinhard Mocek

Ein Kommentar zu diesem Nachdruck des Originalbeitrags ist im Anschluss in diesem Heft und online unter dem DOI: 10.1007/s00048-010-0035-0 zu finden. 
NTM-Schriftenr. Gesch. Naturwiss., Technik, Med., Leipzig 18 (1981) 2, S. 111-123

\section{CHRONIK}

Das diesjährige Gerhard-Harig-Gedenkkolloquium stand unter dem Thema „Von der Universialtät der Wissenschaftsgeschichte“, über das Prof. Dr. sc. phil. $R$. Mocek (Halle) referierte. Im folgenden geben wir den Wortlaut seiner Rede wieder.

Von der Universalität der Wissenschaftsgeschichte

Ein Vortragsthema zu finden, das Gerhard Harigs Intentionen entsprochen haben würde, Problemen unserer heutigen wissenschaftsgeschichtlichen Arbeit gewidmet ist und den Anschluß an die Internationale Theoriediskussion zur Wissenschaftsgeschichte sucht, ist schwer und leicht zugleich. Es ist schwer, weil zu den allgemeinen Anforderungen an das theoretische Niveau unserer Arbeit schon viel gesagt und geschrieben worden ist. Man steht hier irgendwie zwischen Scylla und Charybdis - entweder man ergänzt die Liste der Aufgaben des Wissenschaftshistorikers noch um einige weitere Punkte, dabei wohl wissend, daß das so gut wie überhaupt keinen Zweck hat, aber sehr leicht möglich ist. Oder man reflektiert zum wiederholten Male die Rolle und Bedeutung der Wissenschaftsgeschichte, wozu kaum noch Originelles zu finden sein dürfte. Überdies hat Wissenschaftsgeschichte keine Rechtfertigung mehr nötig - spätestens seit der Zeit, da sie selbst ein Rechtfertigungsinstrument (oder sagen wir besser Legitimationsfaktor) geworden ist. Leicht hingegen ist die Wahl eines solchen übergreifenden Themas mit Blick auf Gerhard Harigs Begriff von Wissenschaftsgeschichte, der dazu geradezu herausfordert. Ich darf vielleicht - ehe ich mich in das Labyrinth des Themas selbst begebe - eine persönliche Erinnerung an meinen Doktorvater Gerhard Harig voranstellen.

Vor fast zwanzig Jahren kam ich zu ihm mit einer Dissertationsthematik, die philosophiegeschichtliche, wissenschaftstheoretische und wissenschaftshistorische Aspekte enthielt. Vereinfacht gesprochen ging es um das Problem der Wechselbeziehung von Philosophie und Biotheorie bei der Herausbildung einer neuen biologischen Disziplin, der Entwicklungsphysiologie tierischer Organismen. Gerade dieses Problem war ja Ende der Fünfziger und Anfang der Sechziger Jahre noch recht belastet. Diffuse Begriffe wie ,,marxistische“ und ,,bürgerliche Naturwissenschaft", eine inhaltlich verstandene Formulierung , Sowjetbiologie“ sowie die Verurteilung biologischer Theorien mit Etiketten wie ,,reaktionärer Mendelismus-Weismannismus-Morganismus" waren entweder noch im Gebrauch oder aber noch nicht weltanschaulich und philosophisch aufgearbeitet und abgeklärt. Seite für Seite blätterte Gerhard Harig meinen Entwurf durch - die vorangegangene Lektüre mußte sehr gründlich gewesen sein. Einen Abschnitt hatte ich mit ,,Erkenntnistheoretische Wurzeln des Idealismus in der Biologie" überschrieben. Das war für mich als Philosophie-Aspirant eine geläufige, weder von mir noch von den damaligen forschenden Philosophen hinterfragte Formulierung. Was sind denn ,,erkenntnistheoretische Wurzeln", fragte er mit der ihm gelegentlich eigenen Bissigkeit, gibt's da auch irgendwo ,,ontologische Blätter" und ,,metaphysisches Astwerk"? Mein schüchtern vorgebrachter Einwand, daß das doch so in den Büchern stünde - ein damals vorherrschendes Argument schnitt er glatt ab mit der Bemerkung, man könnte sich in der Wissenschaft doch wohl ruhig immer mal was Besseres einfallen lassen. Und was soll ,,Idealismus in der Biologie“" Das ist doch auch ein Problem, das man erst einmal untersuchen sollte. Gewiß wurde der Vitalismus von Biologen vertreten - aber gehörte er deshalb zur Wissenschaft der Biologie? Harig forderte Klarheit in theoretischen Dingen, und er forderte auch Theorie für die Wissenschaftsgeschichte. Er ist eigentlich einer der ersten unserer Wissenschaftshistoriker gewesen, die den Begriff der ,,Gesetzmäßigkeiten der Entwicklung der Wissenschaft" als Forschungsaufgabe der Wissenschaftsgeschichte verwendeten, damit die Wissenschaftsgeschichte dazu beitrage, ,,die Planung der wissenschaftlichen Arbeit auf ein sicheres Fundament zu stellen".1

Auf diesen neuralgischen Punkt unserer wissenschaftsgeschichtlichen Arbeiten komme ich noch mehrfach zurück. Und das liegt nicht nur deshalb nahe, weil es Harigs Begriff von Wissenschaftsgeschichte 
entspricht, sondern auch aus dem Grunde, weil es hier um ein Hauptthema der internationalen Wissen. schaftsforschung geht.

Damit möchte ich zunächst vorstellen, zu welchen Fragen ich sprechen werde. Ein einleitender Punkt wird der Diskussionslage zur Frage der Wissenschaftsgeschichte als gesetzesforschender Disziplin gewidmet sein. Das ist tatsächlich der logisch zwingende Einsatz, um die Universalität der Wissenschaftsgeschichte plausibel zu machen. Dann wende ich mich drei Fragen zu, die ich mehr oder weniger andiskutiere, ohne irgenwelchen Anspruch auf Vollständigkeit zu erheben. Es sind folgende Fragen:

1. Können wir Wissenschaftsgeschichte universell bətreiben?

2. Haben wir eine marxistische Theorie der Wissenschaftsgeschichte?

3. Wie stehen wir zum Aufbau bzw. Ausbau einer allgemeinen Wissenschaftsgeschichte?

Zunächst einige Bemerkungen zur Diskussionslage über Gesetzesforschung als Anliegen der Wissenschaftsgeschichte:

In einer 1975 vom Institut für Wissenschaftstheorie und -organisation bei der Akademie der Wissenschaften der DDR vorgelegten Ergebnisübersicht der sowjetischen Literatur zum Erkenntnisstand von Gesetzmäßigkeiten der Wissenschaftsentwicklung im Sozialismus heißt es resümierend - und ich trage dieses Zitat vor, da ich meine, daß sich die Situation seitdem nicht grundlegend geändert hat -, daß ,,zur Problematik spezifischer Entwicklungsgesetzmäßigkeiten der Wissenschaft im Sozialismus ... noch keine ... umfassend ausgearbeitete Theorie" existiert. ${ }^{2}$ Es gibt Vermutungen und Hinweise darauf, daß für die Wissenschaft im Sozialismus neue Entwicklungsgesetzmäßigkeiten entstehen - allein die vielfältigen Formulierungen derartiger Gesetze haben in der Regel der Prüfung nicht standgehalten. Wenn in der problemsuchenden und -definierenden Startphase unserer Wissenschaftstheorie Ende der Sechziger- und Anfang der Siebzigerjahre etwa festgestellt wurde, daß die Gesetzmäßigkeiten, die die Wissenschaftsentwicklung seit der Herausbildung des Kapitalismus bestimmen, erst im Sozialismus voll und allseitig durchgesetzt werden, dann betrifft das wohl einige qualitativ herausragende Wesenszüge von Wissenschaft (Prozeß der Integration, Produktivkraftfunktion), allein wir müssen nüchtern feststellen, daß hier eine spezifische Gesetzmäßigkeit im strengen Wortessinne noch nicht dingfest gemacht wurde. Im Gegenteil : hinsichtlich des produktiven Effekts der unter gegensätzlichen Formationsbedingungen betriebenen Wissenschaft wissen wir heute weniger Sicheres als vor zehn Jahren - damals konnte man noch einige Hypothesen darüber als gesicherte Annahmen betrachten. Mancher leitet daraus die Schlußfolgerung ab, daß sich die Orientierung auf die Erforschung derartiger Gesetzmäßigkeiten wohl nicht bewährt habe. Ich glaube, diese Schlußfolgerung kommt zu früh. Zwei Argumente möchte ich zu bedenken geben. Einmal gibt es in unserer marxistischen Literatur viel zu wenig hypothetische Ansätze, um diese Gesetzmäßigkeiten der Wissenschaftsentwicklung dem historischen Exempel zur Prüfung zu übergeben. Tatsächlich diskutieren wir in dieser Beziehung gegenwärtig Hyopthesen, die innerhalb der nichtmarxistischen Wissenschaftsforschung ausgearbeitet worden sind. Zu nennen wäre Kuhns Paradigmenkonzept, die verschiedenen daran anschließenden Vorschläge zu einer ,,rationalen Rekonstruktion" der Wissenschaftsgeschichte (Lakatoš, Toulmin u. a.) sowie das Finalisierungsmodell der vormaligen Sternberger Gruppe um Böhme, Krohn und van den Daele. Das zweite Argument ist noch stärker auf die heutige Veranstaltung und ihre Träger - die Wissenschaftshistoriker - bezogen: Welche Wissenschaftshistoriker haben denn den Hilferuf der Wissenschaftstheoretiker vor 10 Jahren, sich forschungskonzeptionell an die Analyse der EntwicklungsgesetzmäBigkeiten der Wissenschaft zu wagen, gehört und darauf reagiert? Es ist meines Wissens dazu in der DDR keine wissenschaftshistorische Arbeit erschienen. Und man kann eben nicht erwarten, daß eine so komplizierte Frage ohne die wissenschaftsgeschichtliche Forschung gelöst werden würde. Wir stehen hier vor einem für unsere Wissenschaft insgesamt typischen Kooperationsproblem.

Und damit ist gleich gesagt, daß es hierbei nicht um irgendwelche Vorwürfe und Schuldfragen gehen kann. Hier befinden wir uns auf einer Ebene, die für die wissenschaftliche Gesamtsituation in unserem Lande charakteristisch ist und die erst unlängst wieder Kurt Hager veranlaßte, von einer unbefriedigenden Situation in den Gesellschaftswissenschaften zu sprechen. ${ }^{3}$

In der Mitte der Siebzigerjahre gab es bei uns auch zur Wissenschaftsgeschichte - wohl in Ansehung der beflügelnden Wirkung des VIII. Parteitages der SED auf die gesellschaftswissenschaftliche Selbstbesinnung - eine tiefschürfende Debatte mit nach wie vor zu beherzigenden Anregungen, Kritiken und Vorsätzen für die weitere Arbeit. Stellvertretend für viele Autoren möchte ich die Rede Jürgen Kuczynskis mit dem anspruchsvollen Titel ,,Prolegomena zu einer Geschichte der Wissenschaft" (1974) ${ }^{4}$ und Siegfried Wollgasts Aufsatz ,Zum Verhältnis von Wissenschafts- und Philosophiegeschichte“ (1976)' nennen. An beiden Diskussionsbeiträgen ist so erfreulich, daß man nicht mit allen vorgetragenen Themen einverstanden sein muß nnd dennoch - oder deshalb? - so ungemein viele Anregungen empfängt. Gewiß ist - etwa in Fred Oelßners 
heftiger Erwiderung auf einen anderen, aber ähnlichen Intentionen folgenden Vortrag Kuczynskis ,,Gesellschaftswissenschaftliche Besinnungen" (1973)' sowie in der Akademiedebatte um diese Rede Kuczynskis insgesamt - sofort erkennbar, wo die Skepsis gegenüber einem dargebotenen Legitimations- und Studienfeld ,Wissenschaftsgeschichte“ angesiedelt ist: dort, wo aus der Erinnerung, aus empirischen Versatzstücken, Details und anderen zufällig anmutenden Vergleichen ,Lehren“ für die Gegenwart angeboten werden. ,Wehmütige Rückblicke" nennt Oelßner diese Vorgehensweise", und auch die anderen Redner tolerierten mehr diesen wissenschaftsgeschichtlichen Blick auf Gegenwärtiges, als daß sie ihn akzeptierten.

DieSchwäche der seinerzeit von Kuczynski vorgetragenen Darlegungen besteht - bei aller Sympathie, die ich mit dieser Reflexion hier ausdrücken möchte - darin, daß Kuczynski das Wort und wohl auch die Idee einer Gesetzmäßigkeit der Wissenschaftsentwicklung vermeidet und Wissenschaftsgeschichte damit in ihrer Bedeutung für die Gegenwart auf die Analogie, auf das gute alte Beispiel, auf das Abgucken reduziert. Natürlich konnte auch er nicht die eben gerade dazu vorliegenden Wissenslücken bannen, aber ich glaube, daß die Idee der Gesetzmäßigkeit der Wissenschaftsentwicklung in Kuczynskis Ansatz stärker hervorgehoben werden müßte.

Die sechs Aufgaben, die Kuczynski dem Wissenschaftshistoriker zuweist (Aneinanderreihen von Erkenntnisbausteinen; Ermittlung von Praxisbeziehungen als Ursachen für die jeweilige Wissenschaftsdynamik; Aufweis der methodologischen Mittel, mit denen jeweils Erkenntnisfortschritt erzielt wurde; Untersuchung des Verhältnisses von Weltanschauung und Methodologie; Ermittlung der Prinzipien, die die verschiedenen gesellschaftlichen Klassen der Anwendung der Wissenschaft zugrunde legen; Erforschung all derjenigen Faktoren, die die schöpferische Tätigkeit des Wissenschaftlers in der Vergangenheit erleichtert haben), sind nun allerdings durchaus auf eine ,,gesetzessuchende“" Forschungsorientierung auszubauen - streng genommen ist diese Orientierung aber in den genannten Punkten nicht enthalten. Wollgast stellt diese Frage nun ganz in den Vordergrund. Im Zusammenhang mit der engagierten Kritik an der Beschränkung der Wissenschaftsgeschichte auf bloße Archivstudien stellt Wollgast eine Forderung auf, die logisch zwingend ist: eine marxistische Wissenschaftsgeschichte ist ohne Theorienanspruch undenkbar, Theorieanspruch impliziert die Ermittlung von Gesetzmäßigkeiten, diese sind aber im Kontext einer ganzen geistigen Kultur und vor allem in Verbindung mit dem gedanklichen Gesamtausdruck dieser Kultur - der Philosophie - zu sehen. Für Wollgast ist Wissenschaftsgeschichte ohne Philosophiegeschichte ein Unding - und ich muß gestehen, $\mathrm{da} B$ es mir genauso geht, wobei ich auch die Umkehrung dieser Aussage einschließe: keine Philosophiegeschichte ohne Wissenschaftsgeschichte.

Damit nähern wir uns dem Begriff der ,Universalität“. Seine deutsche Fassung ist relativ einfach - sie lautet ,Allseitigkeit“. Tatsächlich gipfelte die Diskussion um die Aufgaben der Wissenschaftsgeschichte in diesem universellen Anspruch - Geschichte der Wissenschaft ist nicht nur ein sich gesetzmäßig entwickelndes Ganzes, sondern gleichzeitig organischer Bestandteil der materiellen und geistigen Kultur der jeweiligen Epoche, ein Teil der allgemeinen Menschheitsgeschichte. Mikulinskij hat in seiner Einleitung zu der, ,Bibliothek der allgemeinen Geschichte der Naturwissenschaft" - eine vom Institut für Geschichte der Naturwissenschaft und Technik der Akademie der Wissenschaften der UdSSR geplante vielbändige Publikation den Gegenstandsbereich der Wissenschaftsgeschichte nochmals umrissen. Ich möchte das in (nicht autorisierter) Übersetzung wiedergeben: ,Die Entwicklung der Wissenschaft ist direkt und indirekt durch tausende Fäden mit der Entwicklung der Produktion, der ökonomischen, sozialen, ideologischen und politischen Bedingungen, mit dem Kampf der Weltanschauungen, den kultur-historischen Besonderheiten und Traditionen der verschiedenen Völker und Zivilisationen verbunden. Doch widerspiegelt die Wissenschaft die materiellen und geistigen Bedingungen ihrer Zeit nicht passiv. Sie selbst wirkt darauf ein. In mehr oder weniger großem Maße, in Abhängigkeit vom Niveau ihrer eigenen Entwicklung nimmt die Wissenschaft an der Umgestaltung dieser Bedingungen, an der Formierung der Kultur der jeweiligen Perıode teil, indem sie allmählich Gegenstand und Methoden der Erforschung der Umwelt und das Verständnis vom Wesen, den Aufgaben und Zielen der Wissenschaft selbst erweitert und transformiert. Ohne die Analyse dieser vielschichtigen und komplizierten Wechselwirkung kann dor reale Entwicklungsprozeß der Wissenschaft nicht begriffen und adäquat abgebildet werden." 8 Diese Allseitigkeit, d. h. Universalität der Analyse und Synthese wird mit der neuen ,Bibliothek“", deren erster Band mit Rožanskijs Arbeit über die Entwicklung der Naturwissenschaft in der Antike bereits vorliegt, angestrebt. Sind sämtliche ins Auge gefaßten Bände dieser Bibliothek erschienen (zunächst mit 10 Bänden geplant), dann wird nach Mikulinskij ein wesentlicher Beitrag zur weiteren Erarbeitung der Theorie von der Wissenschaftsentwicklung abzurechnen sein!

Und daß es dabei nicht nur um eine platonische Bekundung geht bzw. gehen kann, ist mit der berühmten Bemerkung Lenins im Konspekt zu Hegels ,Wissenschaft der Logik“ untermauert: ,,Die Fortführung des Werkes von Hegel und Marx muß in der dialektischen Bearbeitung der Geschichte des menschlichen Denkens, der Wissenschaft und der Technik bestehen. " 9 Wir entwickeln also die marxistische Dialektik weiter, 
wenn wir diesem Universalitätsanspruch der Wissenschaftsgeschichte folgen. So hoch hängen die Trauben und da ist die Frage berechtigt, ob wir diese Aufgaben nicht denjenigen überlassen sollten, die dafür die besseren Bedingungen und Voraussetzungen haben. Ich glaube, jeder von uns muß sich dieser Frage irgendwie stellen - und ich will es im folgende Punkte tun, den ich überschrieben habe:

\section{Können wir Wissenschaftsgeschichte universell betreiben?}

„Universalität“ heißt natürlich mehr als ,Allseitigkeit“" im quantitativen Sinne. ,Universalität“ hat den Rang einer Kategorie, und zwar in Verbindung mit dem klassischen Bildungshumanismus der deutschen Philosophie des ausgehenden achtzehnten und des beginnenden neunzehnten Jahrhunderts. Dieser Bildungshumanismus zielte auf einen geistigen Kosmos. Wissenschaftliches Wissen bildet danach lediglich das Rohmaterial von Bildung. Der Blick auf das Ganze aber wird durch die Philosophie gegeben. Die Philosophie galt als der Einheitsstifter im System der Wissenschaft, führte wissenschaftliches Wissen jedoch auch zu dem, was man ein ,Ethos“", einen gemeinsamen Sinn nennen kann. Wissenschaft betrieb man, weil man dadurch teilhaftig war an dem Bau dieses geistigen Universums. Auf diese Weise war aber auch die Wissenschaft eingegliedert in eine humanistische Idee. Der Theoriebegriff hatte Bezug zum rechten menschlichen Tun. Nicht von ungefähr spielt die Analyse der Wissenschaft in den Werken der Vertreter der klassischen deutschen Philosophie eine so große Rolle; Fichte ersetzte den Begriff der Philosophie gar durch den Begriff Wissenschaftslehre, nachdem Kant vor ihm die Verläßlichkeit der Vernunft auf die Analyse der menschlichen Erkenntniskräfte gegründet hatte, die ihren vornehmsten Ausdruck in der Wissenschaft finden. Da Kant ziemlich geradlinig Wissenschaft mit Naturwissenschaft identifiziert hatte und diese wiederum auf mathematische Kriterien brachte, fiel aus der Einheit von Philosophie und Wissenschaft allerdings das heraus, was man später die Sozialwissenschaften nannte. Es ist bekannt, daß die Neukantianer die historischen, Kultur- oder Sozialwissenschaften später diesem System als dritte, aber vom an der Naturwissenschaft orientierten Wissenschaftsbegriff unterschiedene Kraft hinzufügten.

Für Hegel war die Geschichte des menschlichen Geistes ein wichtiges Studienfeld für die Ausarbeitung der Dialektik, und Schelling hat die Philosophie direkt definiert als ,Wissenschaft des Wissens". Ich erwähne das hier, weil wir gerade zum Problem des Verhältnisses von Philosophie und Wissenschaft einige historische Verankerungspunkte suchen müssen. Daß uns heute noch Philosophie- und Wissenschaftsgeschichte auseinanderfallen, hat auch in den jeweiligen Etappen der Geschichte der Beziehung beider einen Grund. Aber eben die Einheit von Philosophiegeschichte und Wissenschaftsgeschichte ist ein herausragendes Kriterium einer Universalität von Wissenschaftsgeschichte. Daß wir sie heute schmerzlich vermissen, hat Wollgast überaus plastisch und drastisch klargestellt. Zu Wollgasts Beispielen will ich keine neuen beweisenden Begebenheiten hinzufügen, sondern auf einen Grund der Distanz zwischen beiden Formen des Geschichtsbildes aufmerksam machen. Das hochgesteckte Ziel des einheitlichen Bildungskosmos ist ja nicht aus äußeren Ursachen heraus zusammengebrochen; also nicht etwa deshalb, weil sich kein Bildungssystem fand, das nach den Grundsätzen der klassischen deutschen Philosophie ausbildete. Bekanntlich hatte sich zumindest auf der Ebene herausragender deutscher Gymnasien eine solche Bildungsstätte gefunden. Nein, der geistige Kosmos brach auseinander, weil sich die moderne Naturwissenschaft mit der Einbindung in einen als abgeschlossen geltenden philosophischen Horizont nicht abfinden konnte. Das Buch der Natur war für diese Wissenschaft ausschlaggebend, nicht das Zu-sich-selber-Kommen des objektiven Geistes. Ersteres aber ist - seit Galilei - sachlich und kühl mit mathematischen Formeln, mit Geometrie und Abstraktionen geschrieben letzteres mit dem heißen Atem des Kampfes um den Sieg der Vernunft gegen den Aberglauben, für den wissenden Weg des Subjekts zu Selbstbestimmung und Freiheit. Mathematik und Subjekt-Objekt-Dialektik hatten wieder ganz andere Wege und Ziele für sich bestimmt. Der geistige Kosmos brach auseinander, weil der philosophische Idealismus auf die Dauer die praktisch orientierte Naturwissenschaft nicht in sich bewahren konnte. Der Wissenschaft selbst bekam dieser Ausbruch vorübergehend wohl sehr gut, weniger der Philosophie. Doch auch für die Wissenschaft war die Befreiung von der klassischen deutschen Philosophie keine Befreiung von der Philosophie. Subjekt-Objekt-Dialektik und praktisch orientierte Naturforschung konnten allerdings nur dann wieder auf einen gemeinsamen Nenner kommen, wenn beide im praktischen gesellschaftlichen Lebensprozeß ihre gemeinsame Wurzel entdeckten und sich bewußt machten.

Die Möglichkeit dazu schuf die marxistische Philosophie aufs neue. Für den jungen Marx schon war die Gemeinschaftlichkeit des Anliegens und des, ,Sinnes" von Industrie und Naturwissenschaft mit den Lebensinteressen der tätigen Menschen bereits sonnenklar. Jedoch war das eine wiederhergestellte Einheit in einem, philosophischen Ansatz. In Wirklichkeit hatte sich die Naturwissenschaft verselbständigt. Eine geistige Bindung an eine heroische philosophische Idee gab es in ihr nicht mehr. Spätestens seit Werner von Siemens' programmatischer Verkündung des ,,naturwissenschaftlichen Zeitalters“ auf der Versammlung Deutscher 
Naturforscher und Ärzte im Jahre 1886 fühlte sich die Naturwissenschaft unabhängig von philosophischer Zeitgeisterei und selbst als Produzent des neuen Zeitgeistes. Nach dem Dilemma des ersten Weltkrieges gab es nicht wenige Stimmen, die den ganzen Weg der neueren wissenschaftlichen Zivilisation für verfehlt hielten, hatte sich doch wissenschaftliche und technologische Vernunft in Unvernunft und Destruktivkraft verwandelt. Von einer Klassenanalyse eines imperialistischen Krieges war die übergroße Mehrheit der naturwissenschaftlichen Intelligenz meilenweit entfernt. Ratlosigkeit und Katzenjammer waren zunächst verbreitete Reaktion, ehe sich die vielgeschmähte Philosophie wieder zu Worte meldete und zu einer großangelegten Abrechnung mit der wissenschaftlichen Vernunft ansetzte. Husserl, Horkheimer, Heidegger stellten die Frage, wann die verfehlte Weichenstellung einer auf hektische Weltveränderung zielenden wissenschaftlichen Vernunft denn eingesetzt habe. Diese Weichenstellung datiert - auch für bürgerliches Denken unverkennbar - mit der Herausbildung der bürgerlichen Gesellschaft. Waren also Wissenschaft als Destruktivkraft und bürgerliche Gesellschaft kohärent? Für die genannten Philosophen war diese Einsicht allerdings kein Grund zur durchgreifenden Kritik an dieser Gesellschaft, wenngleich sie angemessen auch erfolgte, sondern eher ein Grund zum abgrundtiefen Pessimismus. Lösungswege - wenn überhaupt wurden im Auseinanderdividieren von Wissenschaft und Denken (die Wissenschaft denkt nicht, ein geflügeltes Wort für Heidegger), in der Verurteilung des bürgerlichen Materialismus, in der Orientierung auf das Abwehrfeld der ,,Innerlichkeit“, des Aushaltens der Entfremdung usw. erblickt. Andere bürgerliche Denker wie $\operatorname{Max}$ Weber betrachteten diese Verirrung des wissenschaftlichen Geistes als ein unabwendbares schicksalhaftes Verhängnis. Auf diese Weise schrieben maßgebliche bürgerliche Philosophen die Leitlinien einer Interpretation der Rolle der modernen Wissenschaft vor - Leitlinien, die in der geschriebenen Wissenschaftsgeschichte natürlich nicht ohne Widerhall blieben. Obwohl hier eine detaillierte Analyse noch aussteht, kann man sagen, daß dieser Einfluß auf die geschriebene Wissenschaftsgeschichte in mehreren Richtungen wirksam wurde. Einmal wurde die historische Folgerichtigkeit des wissenschaftlichen Erkenntnisprozesses in Frage gestellt. Es gebe mehrere mögliche Wege zur Erkenntnis der Welt und des Menschen und der von der , ,abendländischen“ Philosophie und Wissenschaft beschrittene sei einer von mehreren. Wissenschaft wird auf diese Weise als Bestandteil einer, ,diffusen Menge letztendlich gleich irrationaler Theorien“" angesehen. ${ }^{10}$ Diese bei Duhem und Michalski anklingende Argumentation wird konfrontiert durch eine Darstellung, in der in Anlehnung an erste in der nichtmarxistischen Wissenschaftsgeschichtsforschung bekannt werdende sowjetische Arbeiten die Wege der Wissenschaft aus sozialen Faktoren heraus erklärt werden (ohne allerdings die Produktionsverhältnisse zur Kenntnis zu nehmen), wie wir das u. a. bei Borkenau finden. Mumford verfolgt die Geschichte der Wissenschaft in ihrer engen Bindung an Herrschaftsinteresse und einem in der Menschheit irgendwie verfestigten Wahn nach Maschine. Und Crombie schließlich vertritt eine wieder im Ansteigen begriffene Literatur, die die Wissenschaftsgeschichte als ein Alibi des Mystischen, zumindest aber ewig Rätselhaften, das die Menschheit umgebe, deutet. Im Ergebnis einer im einzelnen hervorragenden Analyse der Wechselbeziehung philosophischer und wissenschaftlicher Denkweisen von Augustinus bis Galilei kommt er zu folgendem Resultat: ,Der schöpferische Prozeß originaler Neuentdeckungen und -erfindungen wird immer geheimnisvoll sein, der Einsicht so wenig offen wie die Naturgesetze. Die Geschichte der Wissenschaft vermag uns in philosophischer Erhellung zu zeigen, daß das Denken der großen, bewunderten Erneuerer nach einem anderen Muster geordnet ist als das unsere: Es umgreift einen Komplex von nichtempirischen Konzeptionen und ,regulativen Glaubenssätzen', die - so fremd sie uns sind - Theorien von größter Voraussage- und Erklärungskraft aufzubauen imstande waren. "11 Wissenschaftsgeschichte ist also längst wieder ein Feld der geistig-weltanschaulichen Auseinandersetzung geworden.

Ich kehre zurück zur Ausgangsidee der Frage, ob wir Wissenschaftsgeschichte universell betreiben können. Die Kategorie der Universalität zwingt uns, die Geschichte der Wissenschaft in ihrer Einheit mit der Geschichte des menschlichen Denkens zu sehen. Für Marx war das ein selbstverständlicher Grundsatz. Für den Vertreter der Naturwissenschaft war das keineswegs selbstverständlich. Wie ich zu zeigen versuchte, resultiert diese Haltung aus dem objektiv bedingten Zerfall des humanistischen klassischen Bildungsideals. Die sich von der idealistischen Philosophie lossagende Naturwissenschaft wurde von der marxistischen Philosophie erst relativ spät und vor allem konzeptionell wieder eingeholt. Es kam erst nach und nach zu einer Ausprägung des Bewußtseins, daß der dialektische und historische Materialismus den Rahmen absteckt, in dem die Geschichte und Gegenwart naturwissenschaftlicher und gesellschaftswissenschaftlicher Forschung Platz findet, Platz in einer dialektisch widerstreitenden Einheit der Wissenschaft. Doch man muß wohl darauf hinweisen, daß zwischen der These, wonach der dialektische und historische Materialismus die weltanschauliche Grundlage der modernen Naturwissenschaft bildet, und der Realität, wie dieses Verhältnis tatsächlich funktioniert, Welten liegen können. Auf das von mir diskutierte Problem übertragen heißt das, daß das wissenschaftsgeschichtliche Forschungsfeld philosophisch durchgearbeitet werden 
muß. Diese Aufgabe - und damit verbunden den Vorwurf, daß das so ungenügend geschehen ist - kann man m. E. nun nicht den Fachhistorikern auf dem Gebiet der einzelnen Wissenschaftsgeschichten zuschieben. Sondern das ist eine philosophische Aufgabe.

Fernerhin müssen wir der Tatsache ins Auge sehen, daß die nichtmarxistische Wissenschaftsgeschichtsschreibung zentrale Positionen in den Bibliotheken besetzt hält. Wie wir hierzu stehen, brauche ich nicht lang und breit auszuführen. Wir treten den Verfassern als Fachleuten gegenüber - was wir dabei tun müssen, ist die in der Regel philosophisch begründete Darstellungsidee ihrer Werke genau zu analysieren. Und hier nun liegt des Pudels Kern. Der einen großen marxistischen Gesamtdarstellung stehen Dutzende nichtmarxistische Arbeiten gegenüber. Während die Arbeit Bernals „Die Wissenschaft in der Geschichte“ eben jene eine Gesamtdarstellung - aber ein Vierteljahrhundert marxistischer Forschung einschließlich der gesamten marxistischen Wissenschaftstheorie zwangsläufig nicht enthält (die erste Ausgabe datiert aus dem Jahre 1954), fließen die intensiven Bemühungen nichtmarxistischer Autoren um eine rationale Rekonstruktion der Wissenschaftsgeschichte in viele der neueren nichtmarxistischen Teil- oder Gesamtdarstellungen ein. Ein solcher Vergleich kann einem geradezu die Annahme eines einschlägigen Theoriedefizits der marxistischen Wissenschaftsgeschichte nahelegen.

Und aus diesem Grunde habe ich auch hier die Frage nach der Universalität der Wissenschaftsgeschichte fast ausschließlich auf ihre Einheit mit der Philosophie und Philosophiegeschichte transformiert. Daß Universalität auch bedeutet, einen umfassenden Forschungsansatz zu haben, wie ich es mit dem eingangs zitierten Plan Mikulinskijs demonstriert habe, ist evident. In dieser Beziehung ist die marxistische Wissenschaftsgeschichte universal, weil sie mit dem historischen Materialismus den universellsten Forschungsansatz hat. Das gilt im Prinzip. Das gilt nọch längst nicht für die Praxis unserer Forschungsvorhaben. Wir haben die universellste Wissenschaftsgeschichte, sind aber nicht universell genug. Das soll keineswegs als eine Klage verstanden werden. Ich betrachte es als einen Ansatzpunkt, um über wissenschaftliche Vorhaben nachzudenken. Ein solches Vorhaben wird uns u. a. durch die Diskussion, vor allem die Anregungen des Schlußwortes von Kurt Hager auf der V. Hochschulkonferenz nahegelegt. Den Werdegang des Denkens und seine Höhepunkte in einer Vorlesungsreihe für Hörer aller Fakultäten darzulegen, wissenschaftlich zu zeigen, daß wir aus dem wissenschaftlichen und philosophischen Erbe ableiten das Bewußtsein der geschichtlichen Größe der eigenen Tätigkeit als sozialistische Wissenschaft und Geisteskultur, das ist eine echte Bildungsaufgabe für die Wissenschaftsgeschichte. So werden wir universell, indem wir die Wissenschaftsgeschichte einbeziehen in unseren weltanschaulichen Bildungsauftrag. Universalität zielt auf Theoriedeshalb möchte ich nun einige Gedanken anschließen zu der zweiten Frage, die ich angekündigt hatte:

\section{Haben wir eine marxistische Theorie der Wissenschaftsgeschichte?}

Ich muß hier mit einem Erlebnisbericht beginnen, dessen Anlaß nicht weit zurückliegt. Auf dem 1. Bilateralen Symposium für Pharmaziegeschichte der CSSR und der DDR Anfang Oktober dieses Jahres in Cottbus wurde mir die Ehre zuteil, den Hauptvortrag zu halten. Ich mußte über die Notwendigkeit des Traditionsbewußtseins für den Fortschritt der Wissenschaft sprechen, ein Thema, das theoretisch anspruchsvoll ist. Ich glaube, daß man über das Faktenfeld einer wissenschaftsgeschichtlichen Einzeldisziplin keinen theoretischen Zugang zum Traditionsbegriff findet, daß ein wissenschaftliches 'Traditionsverständnis eine philosophische und wissenschaftstheoretische Angelegenheit ist, die weit mehr impliziert als die bloße Pflege von alten Apothekengegenständen und Ruhestätten ehemals verdienstvoller Apotheker. Die Tagungsthematik war also sehr theoretisch, die Tagungspraxis ausgesprochen pragmatisch. Damit ich nicht mißverstanden werde - mir geht es keineswegs darum, die mühevolle und verdienstvolle faktologische Kleinarbeit der vielen Pharmaziehistoriker, die dort vertreten waren (ich war ehrlich überrascht: nach meiner Ửberschlagsrechnung gibt es weit mehr Pharmaziehistoriker in der DDR als Philosophiehistoriker!!!), geringzuschätzen. Nichts liegt mir ferner als das. Aber ich mußte einfach den Eindruck gewinnen, daß es eine marxistische Theorie der Wissenschaftsgeschichte, die relevant ist auch für die Geschichte einzelner Disziplinen, nicht gibt. Nun wird man einwenden, natürlich gibt es die doch. Da würde ich noch geltend machen, daß eine Theorie, die nicht dort ,,lebt", wo das ihr zugehörige Gegenstandsfeld bearbeitet wird, eben nicht lebt. Mir scheint fast, hier haben wir es wieder mit so einem Fall zu tun, wo wir meinen, prinzipiell ist das Problem gelöst, doch keiner weiß wie. Also etwa hier - prinzipiell ist mit der materialistischen Geschichtsauffassung das Problem einer Theorie der Wissenschaftsgeschichte gelöst.

Wenn dieses ,,im Prinzip ja“" einen Sinn haben soll, dann eigentlich nur, wenn damit eine allgemeine methodologische Orientierung gemeint ist. Dieser methodologischen Orientierung liegt eine Geschichtsphilosophie zugrunde. Und unsere Geschichtsphilosophie, der historische Materialismus, enthält bekanntlich drei Grundforderungen oder Postulate, wie Lenin in einer seiner Schriften vor der Jahrhundertwende feststellte. ${ }^{12}$ Das wäre erstens das Postulat vom gesetzmäßigen Charakter des Geschichtsprozesses, zwei- 
tens das Postulat, wonach die materiellen gesellschaftlichen Bedingungen primär sind gegenüber den ideellen (verkürzt: das gesellschaftliche Sein bestimmt das gesellschaftliche Bewußtsein); drittens das Postulat, daß sich im Längsschnitt und im Querschnitt der Geschichte stets gewisse wiederholbare Strukturen herausbilden, die Produktionsverhältnisse. Deren Etappen bilden die Grundlage für die Periodisierung der Geschichte, die mithin ,,objektiv“" periodisiert ist. Es leuchtet ein, daß diese drei Postulate natürlich nicht hinreichen, um damit auch eine Theorie der Wissenschaft in ihrer Geschichte zu haben. Aber ein methodologisches Fundament dafür sind sie ohne Zweifel. Von diesem Fundament ist nun auszugehen - doch wie und wohin? Soll man nun deduktiv weitere Ansatzpunkte des historischen Materialismus für eine solche Theorie flüssig machen? Also eine deduktive Theorie der Wissenschaftsgeschichte anstreben?

Ich habe an anderer Stelle versucht, ausführlicher zu begründen, weshalb man nicht versuchen sollte, die Theorie einer Einzelwissenschaft deduktiv und gar aus der Philosophie abzuleiten. ${ }^{13}$ Philosophische Grundlagen für eine derartige gesellschaftswissenschaftliche Theorie gibt es gewiß einige und bestimmt noch mehr, als in den drei Postulaten enthalten ist. Doch auch hier ist insofern Vorsicht geboten, als eine vorschnelle Übertragung von Denkmustern aus der Philosophie durchaus auch problematisch sein kann. Ich erinnere hier nur an die lange Zeit, bis wir den einigermaßen festgefahrenen Begriff der Produktivkräfte auch für die Wissenschaft freibekommen haben.

Wenn man sich entscheidet, die Theorie der Wissenschaftsgeschichte im Prozeß der Anwendung der genannten methodologischen Grundlage auf die theoretische Ordnung einzelwissenschaftlichen Materials zu erstreben, dann kämen wir einem Modell von Theorie näher, das auch für andere Wissenschaften gültig ist. Die Voraussetzung dafür aber ist, daß man die Wissenschaftsgeschichte als eine relativ konsistente, einheitliche Gesellschaftswissenschaft anerkennt, nicht aber als eine bloße Summe einzelner Disziplinen. Unsere Frage nach der Theorie der Wissenschaftsgeschichte steht und fällt also mit der Antwort auf die Frage, ob eine allgemeine Wissenschaftsgeschichte nur ein Ordnungswort ist oder aber eine eigene Wissenschaftsdisziplin. Ich entscheide mich dafür, der allgemeinen Wissenschaftsgeschichte den Status einer relativ einheitlichen Wissenschaftsdisziplin zuzuerkennen.

Ich glaube, daß ein Harig-Vortrag ein angemessener Ort und Anlaß ist, um aus all dem abzuleiten, daß wir eine Theorie-Diskussion in unserer Wissenschaftsgeschichte dringend nötig haben. Ich komme zu dieser Annahme auch mit dem Blick auf die Entwicklung der internationalen Wissenschaftsforschung. Auf Mikulinskij hatte ich schon verwiesen. Hier möchte ich noch zwei weitere Quellen nennen: die Studie von Wolfgang Lefèvre: ,Naturtheorie und Produktionsweise. Probleme einer materialistischen Wissenschaftsgeschichtsschreibung - Eine Studie zur Genese der neuzeitlichen Naturwissenschaft" (Darmstadt und Neuwied 1978), und die Arbeit von Peter Dudek: ,Naturwissenschaften und Gesellschaftsformation. Zum Problem der historischen und begrifflichen Konstitution der klassischen bürgerlichen Naturwissenschaften“" (Frankfurt, New York 1979). Wer die quantitativ durchaus nicht überwältigende Diskussion zu den philosophischen Grundlagen der marxistischen Wissenschaftsforschung der letzten zehn Jahre noch in Erinnerung hat, wird bemerken, daß das haargenau die Themen sind, die wir auch diskutiert haben; Naturwissenschaft und Gesellschaftsformation - das ist ein genuin marxistisches Thema. Dudek arbeitet auch mit dem Instrumentarium des historischen Materialismus, jedoch dediziert gegen sowjet-marxistische Interpretationen, wie er sich ausdrückt. Wolfgang Lefèvre, der ehemalige SDS-Vorsitzende in der BRD, legt mit seiner ebenfalls genuin marxistischen Thematik eine auch von der politischen Intention her brauchbare Arbeit vor. Ein Marxist und ein linker Theoretiker in der BRD schreiben zwei beachtliche Bücher über ein Thema, das zu Beginn der Siebzigerjahre in einigen Zeitschriftenaufsätzen bei uns als Forschungsthema vorgelegt wurde. Irgendwie haben wir aber niemals Zeit, derartige Ideen selbst durchzuführen. In der Diskussion zu einer Theorie der Wissenschaftsgeschichte - so hat es ganz den Anschein - haben wir Nachholebedarf nicht nur gegenüber dem eigenen Publikum, sondern auch angesichts der weltweiten Bemühungen zur Erarbeitung einer Theorie der Wissenschaftsgeschichte. Wir sollten in unserer führenden wissenschaftshistorischen Zeitschrift eine Rubrik zu diesem geistigen und weltanschaulichen Problem der Gegenwart eröffnen!

Es geht natürlich nicht darum, daß wir Rückstände gegenüber marxistischen Theoretikern haben, wo wir es nicht vermutet haben. Sondern es geht um inhaltliche Fragen. Die Beziehung von Gesellschaftsformation und Wissenschaft ist die direkte Weiterführung des theoretischen Modells von der zur Produktiv kraft werdenden Wissenschaft. Wenn im Kapitalismus und Sozialismus die Wissenschaft eine vom technologischen Format her gesehen gleichgeartete Produktivkraft-Funktion entfaltet, dann ist die Frage nach den Besonderheiten, die durch die Produktionsverhältnisse in den Wissenschaftsprozeß eingebracht werden und umgekehrt, naheliegend. Aber das ist nicht nur eine rein theoretische, sondern für die Gestaltung des Wissenschaftsprozesses im Sozialismus eminent praktische Frage, die sich - in entsprechender wissenschaftspolitischer Formulierung - auch als die Aufgabe beschreiben läßt, die Vorzüge des Sozialismus mit den Prozessen der wissenschaftlich-technischen Revolution zu verbinden. Wir haben uns in unserem neuen 
Wissenschaftsbereich Wissenschaftstheorie und -geschichte an der Martin-Luther-Universität Halle entschieden, unsere wissenschaftsgeschichtliche Forschung auf diese Thematik zu richten: Wissenschaft und Produktionsverhältnisse in Geschichte und Gegenwart.

Ich möchte nun noch einige abschließende und auch in gewisser Beziehung resümierende Worte zu der dritten angekündigten Frage vortragen:

\section{Wie stehen wir zum Aufbau bzw. Ausbau einer allgemeinen Wissenschaftsgeschichte?}

Bei uns wird gelegentlich und berechtigt das Fehlen einer, ,histoire integrale“ beklagt, das Fehlen einer Geschichtsschreibung, die das jeweils ganze gesellschaftliche Leben umgreift. Die Franzosen haben so etwas, wir erklären es damit, daß sie ja auch keinen Neukantianismus zu verdauen hatten, der die Einheit der jeweils geistigen Kultur theoretisch auseinanderriß. Die vorjährigen Beiträge zum Harig-Gedächtniskolloquium waren bereits diesem Problem gewidmet und ich kann mich auf die dort geäußerten Standpunkte, die Wissenschaftsgeschichte in eine Universalgeschichte einzubeziehen, berufen. Natürlich ist auch das ein Integrationsproblem, und von selbst geschieht da überhaupt nichts. Man kann sich auch nicht darüber hinwegtäuschen, daß ein solches Anliegen außerordentlicher Anstrengung bedarf; und mancher von uns hat niemals die Möglichkeit gehabt, sich in dem erforderlichen breiten Maße auf eine solche Aufgabe vorzubereiten. Das ist eine echte Herausforderung für den wissenschaftlichen Nachwuchs und bedarf sehr langfristiger Planung. Obwohl ich meine, daß das eine Aufgabe für unseren Arbeitskreis ist, will ich darauf nicht näher eingehen, denn zu solchen Problemen sollte man nicht monologisieren, sondern streiten.

Ich möchte stattdessen stichwortartig verdeutlichen, warum man auf eine allgemeine Wissenschaftsgeschichte nicht verzichten kann, wenn man Wissenschaftsgeschichte tatsächlich und ernsthaft als Einheit der seinerzeit von Mikulinskij und Rodnyi geforderten vier Ebenen akzeptiert: Archivarbeit, beschreibende Arbeiten, analytische Arbeiten, schließlich Untersuchungen, die die Wissenschaftsentwicklung im Zusammenhang mit dem Geschichtsverlauf und den großen ideellen und kulturellen Strömungen verfolgen. ${ }^{\mathbf{1 4}}$

Erstens: Es gibt keine Entwicklungsgesetze der Wissenschaft, die nur für eine Wissenschaftsdisziplin in der Geschichte gelten. W'issenschaftsgeschichte als gesetzesermittelnde Disziplin muß in einer allgemeinen Wissenschaftsgeschichte zusammengefaßt sein.

Zweitens: Naturtheoretische und sozialtheoretische Überlegungen waren in der Geschichte der Wissenschaft die weitaus größten Zeitabschnitte über aufeinander bezogen. Noch die klassischen englischen Ökonomen verstanden sich nicht als Volkswirtschaftler, sondern als Universalwissenschaftler, verstanden eine Menge von der Naturwissenschaft und Mathematik ihrer Zeit. Die Trennung von Naturwissenschaft und Gesellschaftswissenschaft datiert erst aus jüngster Zeit. Wir übergehen diese Tatsache oft damit, daß wir sagen, die Gesellschaftswissenschaft habe sich ja erst vor einhundert Jahren herausgebildet. So gesehen, gibt es auch erst eine Philosophie, Ethik, Ökonomie usw. seit einhundert Jahren, was offensichtlich nicht stimmt.

Drittens: Eine geistige Kultur wird in der Regel durch ein wissenschaftliches Gesamtpotential, durch weltanschauliche Ideen, die dieses Potential durchwirken, durch eine allgemeine objektive Anforderungssituation an die Wissenschaft, durch übereinstimmende soziologische und ideologische Aspekte der Arbeit der Wissenschaftler charakterisiert. Untrennbar damit verbunden ist ein bestimmter Einfluß herrschender Moral und ein geistiger Kontakt zur Kunst. Diese Elemente bilden, zusammen mit der herrschenden Ideologie, eine im Grunde genommen untrennbare Ganzheit. Wenn der Begriff nicht so abgegriffen wäre, würde ich sagen, daß wissenschaftsgeschichtliche Untersuchungen sich auch eingliedern müßten in das, was man die ,,geistigen Strömungen“ einer Epoche, einer Formation, einer Kultur, eines Landes nennt. Wissenschaft ist ja nie das Ganze einer geistigen Kultur. Aber sie hat Valenzen zu alledem, sie ist selbst allseitig, universell. So sollte auch ihr geschichtliches Bild sein.

\section{Anmerkungen}

1 G. Harig: Aspekte der Geschichte der Naturwissenschaft, in : NTM 3 (1966) 44.

${ }^{2}$ H. Fetzer: Zum Erkenntnisstand von Gesetzmäßigkeiten der Wissenschaftsentwicklung im Sozialismus, Inst. f. Wiss.theorie u. -org. der AdW d. DDR, Studien und Forschungsberichte, 6 (1975) 71.

${ }^{3}$ K. Hager: Schlußwort auf der V. Hochschulkonferenz, ND v. 6./7. 9. 80.

4 J. Kuczynski: Prolegomena zu einer Geschichte der Wissenschaft, Sitzungsberichte der AdW der DDR, 4/1974, Berlin 1974 . 
5 S. Wollgast: Zum Verhältnis von Wissenschafts- und Philosophiegeschichte, in: Wiss. Zeitschr. d. Humboldt-Univ. Berlin Ges.- u. Sprachwiss. Reihe, XXV (1976) 1, S. 37-42.

${ }^{6}$ J. Kuczynski: Gesellschaftswissenschaftliche Besinnungen, Sitzungsberichte des Plenums und der Klassen der AdW der DDR 7 (1973) Akademie-Verlag Berlin 1973.

7 Diskussionsbeitrag $F$. Oelßner in : ebenda, S. 38.

${ }^{8}$ S. R. Mikulinskij: Einleitung zur Serie: Bibliothek der allgemeinen Geschichte der Naturwissenschaft, in: $J$. D. Rožanskij: Die Entwicklung der Naturwissenschaft in der Antike. Die frühe griechische Wissenschaft von der Natur, Moskau 1979 (Arbeitsübersetzung v. R. Peuckert)

9 W. I. Lenin: Konspekt zu Hegels,,Wissenschaft der Logik“", in: Werke, Bd. 38, Berlin 1964, S. 137.

${ }^{10}$ Vgl. W. Lefèvre: Naturtheorie und Produktionsweise, Darmstadt und Neuwied 1978.

${ }^{11}$ A. C. Crombie: Von Augustinus bis Galilei. Die Emanzipation der Naturwissenschaft, Köln, Berlin (West) 1959, S. 561 der Taschenbuchausgabe München 1971.

12 W. I. Lenin: Was sind die ,,Volksfreunde“" und wie kämpfen sie gegen die Sozialdemokraten?, in: Lenin, Werke Bd. 1, Berlin 1972.

${ }^{13} \mathrm{Vgl}$. R. Mocek: Gedanken zur Wissenschaft, Berlin 1980.

${ }^{14}$ S. R. Mikulinskij, N.I. Rodnyi: Wissenschaftsgeschichte und Wissenschaftskunde, in: WissenschaftStudien zu ihrer Geschichte, Theorie und Organisation, Berlin 1972, S. 63 f.

Prof. Dr. sc. phil. Reinhard Mocek

Martin-Luther-Univ. Halle-Wittenberg Wissenschaftsbereich Wissenschaftstheorie und -geschichte 